\title{
CLASSIFICAC̄̃̃ DE RECURSOS MINERAIS USANDO A VARIÂNCIA DE INTERPOLAÇÃO
}

\author{
JORGE KAZUO YAMAMOTO* \& RITA PARISI CONDE**
}

\begin{abstract}
ORE RESOURCE CLASSIFICATION USING THE INTERPOLATION VARIANCE This paper presents a proposal for mineral resources classification using the interpolation variance. This alternative for measuring the uncertainty associated with the ordinary kriging estimate allows characterization of the local dispersion of data, as well as recognition of the proportional effect when it is present. Therefore, the interpolation variance presents the main characteristics for quantification of uncertainty and consequently it can be used for mineral resources classification. The tolerance error, calculated with the interpolation variance, is used to establish certainty classes, according to a classification model widely accepted in the mineral industry. The proposed model was tested for $\mathrm{Pb}$ and $\mathrm{Zn}$ resources classification for Canoas 2 Mine. According to the results, the classification provided by the interpolation variance is more reliable than that provided by kriging variance. It is important to note that the tolerance error leveis adopted for mineral resources classes will not allow classification of ali mineral deposits, mainly those that present high natural variability. However, the proposed methodology quantifies the tolerance errors, which are important for the knowledge of the deposit, as well as for mine planning.
\end{abstract}

Keywords: Interpolation variance, kriging variance, ordinary kriging, ore resource classification, Canoas 2 Mine

RESUMO Este trabalho apresenta uma proposta para a classificação de recursos minerais usando a variância de interpolação. Esta alternativa para medida da incerteza associada à estimativa por krigagem ordinária permite caracterizar a dispersão local dos dados, bem como reconhecer o efeito proporcional, quando existente. Portanto, a variância de interpolacão reúne as características essenciais para quantificação da incerteza e, assim, pode ser usada para fins de classificacão de recursos minerais. O erro de tolerância, calculado com base na variância de interpolação, é usado para estabelecer classes de certeza, conforme um modelo de classificação largamente aceito na indústria mineral. O modelo proposto foi testado para classificação de recursos de $\mathrm{Pb}$ e $\mathrm{Zn}$ da Mina de Canoas 2 . Conforme os resultados obtidos, a classificação proporcionada pela variância de interpolação é mais confiável que a pela variância de krigagem. Cabe ressaltar, contudo, que os níveis de erros de tolerância adotados para as classes de recursos minerais não permitirão classificar todos os depósitos minerais, principalmente aqueles que apresentam alta variabilidade natural. Entretanto, a metodologia proposta permite quantificar os erros de tolerância, os quais são importantes tanto para o conhecimento do depósito, bem como para fins de planejamento da lavra.

Pa/avras-c/zaveí.-variância da interpolação, variância da Krigagem, Krigagem ordinária, classificação de recursos minerais

INTRODUÇÃO A aplicação de métodos geoestatísticos para o cálculo de reservas minerais tem sido, por várias razões, aceita como padrão da indústria mineral. As principais razões são o conhecimento do depósito ou da variabilidade dos dados por meio da análise do variograma e, numa etapa posterior, a estimativa das variáveis de interesse por meio da krigagem. Krigagem é o nome genérico dado a um conjunto de técnicas de regressão linear generalizada que minimiza a variância de estimativa, definida a partir de um modelo de covariância (Olea 1991). Além destas razões, estas técnicas contribuíram para o sucesso dos métodos geoestatísticos, justamente pela possibilidade de quantificar o erro associado à estimativa através da variância de krigagem.

A variância de krigagem foi utilizada por muitos autores para aferir a qualidade da estimativa, bem como para a classificação de reservas minerais. Contudo, vários autores descobriram que a variância de krigagem não poderia ser aplicada como medida real de dispersão (Journel 1986, Olea 1991, Yamamoto 1991), pois não levava em consideração a variabilidade local dos dados. Assim, a variância de krigagem não pode ser utilizada para determinação do erro de estimativa para fins de classificação de reservas minerais (Armstrong 1994).

Na realidade, a variância de krigagem apresenta a propriedade de homoscedasticidade (Olea 1991), isto é, independente da variabilidade do conjunto de dados utilizado para realizar a estimativa. Por estes motivos, este autor vem pesquisando o assunto e propondo alternativas para uma medida de incerteza associada à krigagem ordinária (Yamamoto 1991 e 2000). Esta alternativa foi denominada variância de interpolação e é baseada na média ponderada das diferenças ao quadrado entre os valores observados e a estimativa calculada. A variância de interpolação mede a dispersão local dos pontos de dados, bem como reconhece o efeito proporcional quando existente (distribuições com assimetria positiva). Portanto, a variância de interpolação surge como uma alternativa viável à quantificação da incerteza associada à estimativa de teores e, dessa forma, pode ser aplicada para fins de classificação de reservas minerais.

Este trabalho apresenta uma revisão sobre as medidas de variância proporcionadas pela geoestatística, bem como uma fundamentação teórica da variância de interpolação e sua relação com a variância de krigagem. Neste trabalho, propõe-se um modelo de classificação de recursos minerais usando a variância de interpolação para determinar o erro de estimativa. Por fim, o modelo é aplicado para classificação de recursos de $\mathrm{Pb}$ e Zn na Mina de Canoas 2, Adrianópolis, PR.

\section{NOMENCLATURA E DEFINICÕES A variância de exten-} são, de acordo com Matheron (1971), mede a extensão de uma única amostra em sua zona de influência. A estimativa pelo método dos polígonos (estimativa pela amostra mais próxima) está afetada pela variẩncia de extensão (Fig. IA).

Segundo Matheron (1971), quando mais de uma amostra são usadas para fazer uma estimativa, o erro destas múltiplas extensões de um grande número de amostras é definido como variância de estimativa (Fig. 1B). Genericamente, a variância de estimativa é a variância do erro de estimativa (Olea 1991).

A variância de krigagem de uma função aleatória $\mathrm{Z}(\mathrm{x})$, de acordo com Olea (1991), é definida como variância de estimativa não condicional $E\left[Z(X o)-Z^{*}\left(X_{0}\right)\right]^{2}$ resultante de um processo de krigagem linear (englobando krigagem simples, ordinária e universal). Entre as várias formas de krigagem, a krigagem ordinária $(\mathrm{KO})$ tem sido largamente utilizada como um método de estimativa confiável e, portanto, será considerada como estimador da krigagem. Embora os ponderadores da krigagem ordinária sejam resultantes da minimização da variância de estimativa, este termo geral deveria ser reservado para designar uma variância correspondente à dispersão em torno de uma estimativa resultante de qualquer método de estimativa, seja ele krigagem ou não.

VARIÂNCIA DE KRIGAGEM A estimativa do valor da variável de interesse em um ponto não amostrado pode ser obtida como:

$$
\mathbf{Z}^{*}\left(\mathbf{x}_{n}\right)=\sum_{\mathbf{1}=1}^{\dot{M}} \lambda_{1} \mathbf{Z}\left(\mathbf{x}_{1}\right)
$$

onde $\left\{\lambda_{i}, \hat{j}=1, n\right\}$ são os ponderadores da krigagem ordinária, resultantes da minimização da função variância do erro, sujeitos à condição de não enviesame $\sum_{i=1}^{a} \lambda_{1}=1$

\section{A variância do erro é:$$
\sigma_{\text {ko }}^{2}=\operatorname{Var}\left(Z\left(\mathbf{X}_{\mathrm{o}}\right)-\mathbf{Z}^{*}\left(\mathbf{X}_{\mathrm{o}}\right)\right\} \text {, }
$$

que pode ser desenvolvida como:

$$
\sigma_{k o}^{2}=\operatorname{Cov}\left(Z\left(X_{0}\right) Z\left(X_{0}\right)\right\}-2 \operatorname{Cov}\left\{Z^{*}\left(X_{0}\right) Z\left(X_{0}\right)+\operatorname{Cov}\left\{Z^{*}\left(X_{0}\right) Z^{*}\left(X_{0}\right)\right\}\right)
$$
cada termo do lado direito desta expressão pode ser escrito como:

$$
\operatorname{Cov}\left\{\mathbf{Z}\left(\mathbf{X}_{\mathrm{o}}\right) \mathbf{Z}\left(\mathbf{X}_{\mathrm{o}}\right)\right\}=\operatorname{Var}\left\{\mathbf{Z}\left(\mathbf{x}_{\mathrm{u}}\right)\right\}=\mathbf{C}(0)
$$

* Departamento de Geologia Sedimentar e Ambiental, Instituto de Geociências, Universidade de São Paulo. Caixa Postal 11.348, CEP 05.422-970, São Paulo, Brasil.E-mail: jkyamamo@usp.br.

** Programa de Pós-Graduacão em Recursos Minerais e Hidrogeologia, Instituto de Geociências, Universidade de São Paulo. Caixa Postal 11.348, CEP 05422-970, São Paulo, Brasil. E-mail: rconde@usp.br. 
A

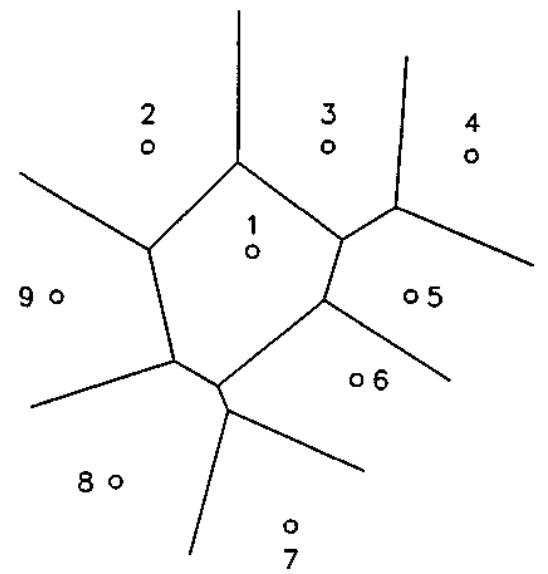

B

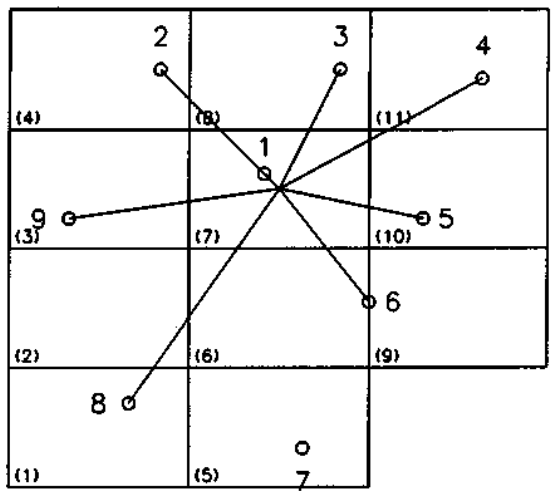

Figura l - Conceitos de variância de extensão, erro cometido pela extensão do teor da amostra 1 no poligono de influência (A) e da variância de estimativa, devido às múltiplas extensões para cálculo do teor no bloco de cubagem 7 (B). Pontos de dados, segundo Popoff(1966).

$$
\begin{aligned}
& 2 \operatorname{Cov}\left\{Z^{*}\left(\mathbf{x}_{0}\right) \mathbf{Z}\left(\mathbf{x}_{0}\right)\right\}=2 \operatorname{Cov}\left\{\left[\sum_{1} \lambda_{1} \mathbf{Z}\left(\mathbf{x}_{1}\right)\right] \mathbf{Z}\left(\mathbf{x}_{\mathbf{0}}\right)\right\} \\
& =2 \mathbf{E}\left\{\sum_{1} \lambda_{1} Z\left(x_{1}\right) \mathbf{Z}\left(x_{0}\right)\right\}-2 \mathbf{E}\left\{\sum_{1} \lambda_{1} Z \mathbf{Z}\left(x_{i}\right)\right\} \mathbf{E}\left\{\mathbf{Z}\left(\mathbf{x}_{\mathbf{o}}\right)\right\} \\
& =2 \sum_{i} \lambda_{1} \mathbf{E}\left\{\mathbf{Z}\left(\mathbf{x}_{1}\right) \mathbf{Z}\left(\mathbf{x}_{\mathrm{v}}\right)\right\}-2 \sum_{i} \lambda_{1} \mathbf{E}\left\{\mathbf{Z}\left(\mathbf{x}_{\mathbf{i}}\right)\right\} \mathbf{E}\left\{\mathbf{Z}\left(\mathbf{x}_{\boldsymbol{v}}\right)\right\} \\
& =2 \sum_{i} \lambda_{1}\left[\mathbf{E}\left\{\mathbf{Z}\left(\mathbf{x}_{1}\right) \mathbf{Z}\left(\mathbf{x}_{0}\right)\right\}-\mathbf{E}\left\{\mathbf{Z}\left(\mathbf{x}_{i}\right) \mathbf{E}\left\{\mathbf{Z}\left(\mathbf{x}_{0}\right)\right\}\right\}\right] \\
& =2 \sum_{1} \lambda_{1} \mathrm{C}\left(\mathbf{x}_{\mathbf{i}}-\mathbf{x}_{0}\right) \\
& \operatorname{Cov}\left\{\mathbf{Z}^{*}\left(\mathbf{x}_{\mathbf{o}}\right) \mathbf{Z}^{*}\left(\mathbf{x}_{\mathbf{0}}\right)\right\}=\operatorname{Var}\left\{\mathbf{Z}^{*}\left(\mathbf{x}_{\mathrm{o}}\right)\right\} \\
& =\operatorname{Var}\left\{\sum_{1} \lambda_{1} \mathbf{Z}\left(x_{1}\right)\right\} \\
& =\sum_{i} \sum_{j} \lambda_{1} \lambda_{j} \mathbf{C}\left(\mathbf{x}_{\mathrm{i}}-\mathbf{x}_{\mathrm{j}}\right)
\end{aligned}
$$

Portanto, a variância do erro pode ser escrita como:

$$
\sigma_{\mathrm{Ko}}^{2}=\mathbf{C}(0)-2 \sum_{1} \lambda_{1} \mathrm{C}\left(\mathbf{x}_{1}-\mathbf{x}_{0}\right)+\sum_{1} \sum_{j} \lambda_{1} \lambda_{j} \mathbf{C}\left(\mathbf{x}_{1}-\mathbf{x}_{j}\right)
$$

Para encontrar o mínimo da função variância do erro, sujeita à

$$
L\left(\lambda_{1}, \lambda_{2}, \cdots, \lambda_{\mu}, \mu\right)=C(0)-2 \sum_{i} \lambda_{t} C\left(x_{i}-x_{v}\right)+\sum_{i} \sum_{i} \lambda_{i} \lambda_{j} c\left(x_{i}-x_{j}\right)-2 \mu\left(\sum_{i} \lambda_{i}-1\right)
$$

condição de restrição, forma-se o Lagrangiano:

Cabe observar que Isaacks \& Srivastava (1989) ao desenvolverem

$$
\frac{d L}{d \lambda_{i}}=-2 C\left(x_{i}-x_{o}\right)+2 \sum_{j} \lambda_{j} C\left(x_{i}-x_{j}\right)-2 \mu=0
$$

para $\mathrm{i}=1, \mathrm{n}$,

$$
\frac{d L}{d \mu}=\sum_{i} \lambda_{j}-1=0,
$$

e a derivada $\mathrm{dL} / \mathrm{d} \mu$ também é igualada a zero:

$$
\left\{\begin{array}{l}
\sum_{j} \lambda_{j} C\left(x_{i}-x_{j}\right)-\mu=C\left(x_{i}-x_{0}\right) \text { para } i=1, n \\
\sum_{j} \lambda_{j}=1
\end{array}\right.
$$

o Lagrangiano, somam à função objetivo (equação 5), ao invés de subtraírem, o parâmetro de Lagrange $(2 \mu)$ multiplicado pela condição de não enviesamento. Para encontrar o mínimo do Lagrangiano, determina-se as derivadas parciais $\mathrm{dL} / \mathrm{d} \lambda_{\mathrm{i}}$ e igualadas a zero, como segue:

Observe-se que em Isaacks \& Srivastava (1989), no sistema de equações de krigagem, escrito em termos das covariância, o parâmetro de Lagrange aparece com o sinal trocado. As primeiras n equações do sistema (6), multiplicadas por $\lambda_{\mathrm{i}} \mathrm{e}$ somadas $\mathrm{n}$ vezes, ficam:

$$
\sum_{i} \sum_{j} \lambda_{i} \lambda_{j} C\left(x_{i}-x_{j}\right)-\mu=\sum_{j} \lambda_{i} C\left(x_{i}-x_{o}\right)
$$

que substittídas em (5) resultam na variância de krigagem ordinária:

$$
\sigma_{k o}^{2}=C(0)-\sum_{j} \lambda_{j} C\left(x_{i}-x_{o}\right)+\mu
$$

A variância de krigagem pode ser escrita também em termos da função variograma, tendo em vista que $C(h)=\mathrm{C}(\mathrm{o})-\gamma(h)$ :

$$
\begin{aligned}
\sigma_{k o}^{2} & =\sum_{i} \lambda_{j} \gamma\left(x_{i}-x_{o}\right)+\mu \\
S_{o}^{2} & =\sum_{i} \lambda_{i}\left[Z\left(x_{i}\right)-Z^{*}\left(x_{o}\right)\right]^{2}
\end{aligned}
$$

persão associada à estimativa $Z^{*}\left(\mathrm{x}_{\mathrm{O}}\right)$ é calculada como:

$$
S^{2}=\sum_{i} P\left(Z\left(x_{i}\right)\right)\left[Z\left(x_{i}\right)-Z^{*}\left(x_{o}\right)\right]^{2}
$$

onde $\mathrm{P}(\mathrm{Z}(\mathrm{Xj}))$ é a probabilidade de ocorrência da variável $\mathrm{Z}\left(\mathrm{X}_{\mathrm{i}}\right)$ e considerada igual a $1 / n$, assumindo-se a hipótese de independência entre as $\mathrm{n}$ amostras. A variância de interpolação, proposta originalmente por Yamamoto (1991), é obtida substituindo-se a probabilidade de ocorrência $\mathrm{P}(\mathrm{Z}(\mathrm{Xj}))$ pelo ponderador da krigagem ordinária ${ }^{\wedge}$

Na realidade, o conjunto de ponderadores $\left\{\lambda_{j} \mathrm{i}=, \mathrm{n}\right\}$ é interpretado como uma distribuição de probabilidades, pois $\Sigma$

Contudo, como se sabe, a krigagem ordinária não garante que todos os ponderadores sejam positivos, o que faz com que, sob algumas condições, ocorram pesos negativos. Estas condições são a ocorrência de agrupamento de pontos e a utilização de variograma com alto grau de continuidade na origem. Atualmente, existem algoritmos eficientes para a eliminação de pesos negativos. A título de illustração, pode-se citar aqueles propostos por Froidevaux (1993), Journel \& Rao (1996) e Deutsch (1996), que consistem no pós-processamento dos pesos resultantes, analisando e eliminando os pesos negativos e recalculando os pesos positivos para que a soma seja igual a 1. Portanto, os pesos negativos podem ser eliminados e os pesos corrigidos aplicados à expressão (10) garantem uma variância de interpolação sempre positiva.

Segundo Yamamoto (2000), a variância de interpolação apresenta as seguintes características:

- corresponde à propriedade de exatidão da $\mathrm{KO}$, isto é, se o ponto a ser estimado coincide com um ponto de dado, então o peso deste ponto é igual a um com todos os outros pesos iguais a zero, portanto $\mathrm{S}_{0}^{2}=0$

- é proporcional à dispersão dos pontos de dados;

- usa indiretamente a distância estrutural do variograma através do peso da KO $\lambda_{i}$. Quanto mais influente o ponto de dado maior o seu peso.

A variância de interpolação pode ser desenvolvida da seguinte forma:

$$
\begin{aligned}
S_{o}^{2} & =\sum_{i} \lambda_{i} Z^{2}\left(x_{i}\right)-2 Z^{*}\left(x_{o}\right) \sum_{i} \lambda_{i} Z\left(x_{i}\right)+Z^{* 2}\left(x_{o}\right) \\
& =\sum_{i} \lambda_{i} Z^{2}\left(x_{i}\right)-Z^{* 2}\left(x_{o}\right)
\end{aligned}
$$


Subtraindo a esperança matemática, $\mathrm{m}=\mathrm{E}[\mathrm{Z}(\mathrm{x})]$, das duas variáveis aleatórias $Z(x i)$ e $Z^{*}(x p)$ e elevando-se ao quadrado, a expressão da variância de interpolação permanece inalterada:

$$
S_{o}^{2}=\sum \lambda_{i}\left[Z\left(x_{j}\right)-m\right]^{2}+\sum\left[Z^{*}\left(x_{o}\right)-m\right]^{2}
$$

Aplicando-se o operador da esperança matemática em ambos os lados da expressão da variância de interpolação tem-se:

$$
\begin{gathered}
E\left[S_{o}^{2}\right]=\sum_{i} \lambda_{i} E\left[Z\left(x_{i}\right)-m\right]^{2}+E\left[Z^{*}\left(x_{o}\right)-m\right]^{2} \\
=\sum_{i} \lambda_{i} \operatorname{Var}\left[Z\left(x_{i}\right)\right]+\operatorname{Var}\left[Z^{*}\left(x_{o}\right)\right] \\
=\operatorname{Var}\left[Z\left(x_{i}\right)\right]-\operatorname{Var}\left[Z^{*}\left(x_{o}\right)\right]
\end{gathered}
$$

Substituindo-se (2) e (4) em (1 1) tem-se:

$$
\begin{aligned}
E\left[S_{o}^{2}\right] & =C(0)-\sum_{i} \sum_{j} \lambda_{i} \lambda_{j} c\left(x_{i}-x_{j}\right) \\
& =\sum_{i} \sum_{j} c(0)-\sum_{i} \sum_{j} c\left(x_{i}-x_{j}\right) \\
& =\sum_{i} \sum_{j} \lambda_{i} \lambda_{j}\left[C(0)-C\left(x_{i}-x_{j}\right)\right]
\end{aligned}
$$

A expressão (5), após adição e subtração do termo $\sum_{i} \sum_{j} \lambda_{i} \lambda_{j} c\left(x_{i}-x_{j}\right)$, pode ser rescrita como:

$$
\begin{aligned}
\sigma_{x o}^{2}= & \sum_{i} \sum_{j} \lambda_{i} \lambda_{j} C(0)-\sum_{i} \sum_{j} \lambda_{i} \lambda_{j} C\left(x_{i}-x_{j}\right)+ \\
& +2 \sum_{i} \sum_{j} \lambda_{i} \lambda_{j} C\left(x_{i}-x_{j}\right)-2 \sum_{i} \lambda_{i} C\left(x_{i}-x_{o}\right) \\
= & \sum_{i} \sum_{j} \lambda_{i} \lambda_{j}\left[C(0)-C\left(x_{i}-x_{j}\right)\right]+ \\
& +2 \sum_{i} \lambda_{i}\left[\sum_{j} \lambda_{j} C\left(x_{i}-x_{j}\right)-C\left(x_{i}-x_{o}\right)\right]
\end{aligned}
$$

Nesta expressão, o primeiro termo é igual a $E\left[S_{0}^{2}\right]$, de acordo com a equação (12) e, conforme a equação (7), o valor entre colchetes do segundo termo é igual a $\mu$. Assim, a expressão (13) fica:

$$
\begin{aligned}
\sigma_{k o}^{2} & =E\left[S_{o}^{2}\right]+2 \sum_{i} \lambda_{j} \mu \\
& =E\left[S_{o}^{2}\right]+2 \mu
\end{aligned}
$$

ou

$$
E\left[S_{o}^{2}\right]=\sigma_{K O}^{2}-2 \mu
$$

A esperança matemática da variância de interpolação é igual à variância de krigagem subtraída de duas vezes o multiplicador de Lagrange. Nesta expressão tem-se a explicação por que a variância de krigagem mede apenas a configuração espacial dos pontos de dados e não a dispersão local. Observe-se que as dispersões locais, medidas por meio da variância de interpolação, foram suavizadas através do operador da esperança matemática.

Eliminação de pesos negativos Como introduzido anteriormente, os pesos negativos resultantes da solução do sistema de equações de krigagem (6) podem causar variância de interpolação negativa. Contudo, deve-se salientar que, principalmente em distribuições lognormais, a utilização de pesos negativos pode resultar em estimativas negativas que, no caso de estimativa de teores, densidades e espessuras, são inadmissíveis. O usuário de programas de cálculo de reservas baseados na técnica da krigagem ordinária já deve ter deparado com este problema, que é comum, quando a variável de interesse segue uma distribuição lognormal.

Existem basicamente dois tipos de algoritmos para solução do problema de pesos negativos da krigagem ordinária: o primeiro trabalha sobre o sistema de equações garantindo que todos os pesos sejam positivos (Herzfeld 1989, Barnes \& Johnson 1984) ou, então, aqueles que tratam diretamente os pesos negativos, eliminando-os (Froidevaux 1993) ou eliminando e transladando-os (Journel \& Rao 1996). Neste trabalho, serão considerados aqueles algoritmos que trabalham diretamente sobre os pesos negativos, tais como o de Froidevaux (1993) e o de Journel \& Rao (1996).
O algoritmo de Froidevaux (1993) iguala a zero os pesos negativos e recalcula os pesos restantes para que a soma dos pesos seja igual a um:

$$
\left\{\begin{array}{l}
\text { Para } i=1, n \text { se } \lambda_{i}<0 \Rightarrow \lambda_{i}=0 \\
\tau_{i}=\frac{\lambda_{i}}{\sum \lambda_{j}}
\end{array}\right.
$$

onde $\left\{\lambda_{i} i=1, n\right\}$ são os pesos recalculados e utilizados para a realização da estimativa (1), cálculo da variância de krigagem (8) e da variância de interpolação (10).

Journel \& Rao (1996) propuseram o seguinte algoritmo:

$$
\left\{\begin{array}{l}
c=-\min \left(\lambda_{i}, i=1, n\right) \\
\tau_{i}=\frac{\lambda_{i}+c}{\sum_{j}\left(\lambda_{j}+c\right)}
\end{array}\right.
$$

onde e é uma constante igual ao maior peso negativo em módulo, que é adicionada a todos os pesos obtidos da $\mathrm{KO}$ e recalculados para que a soma seja igual a um.

A diferenca entre estes algoritmos está no número de amostras que são eliminadas da estimativa. Froidevaux (1993) elimina todas as amostras com pesos negativos, enquanto Journel \& Rao (1996) eliminam somente a amostra com o maior peso negativo em módulo.

\section{AVALIAÇÃO DE RESERVAS EM BLOCOS DE CUBAGEM}

Como se sabe, a geoestatística proporcionou a ferramenta para a avaliação de blocos de cubagem denominada krigagem ordinária de bloco ou simplesmente krigagem de bloco. Ao invés de estimar o bloco usando apenas o ponto central (Fig. 2), o bloco pode ser estimado a partir de sua subdivisão em sub-blocos (Fig. 3), o que melhora em muito a estimativa.

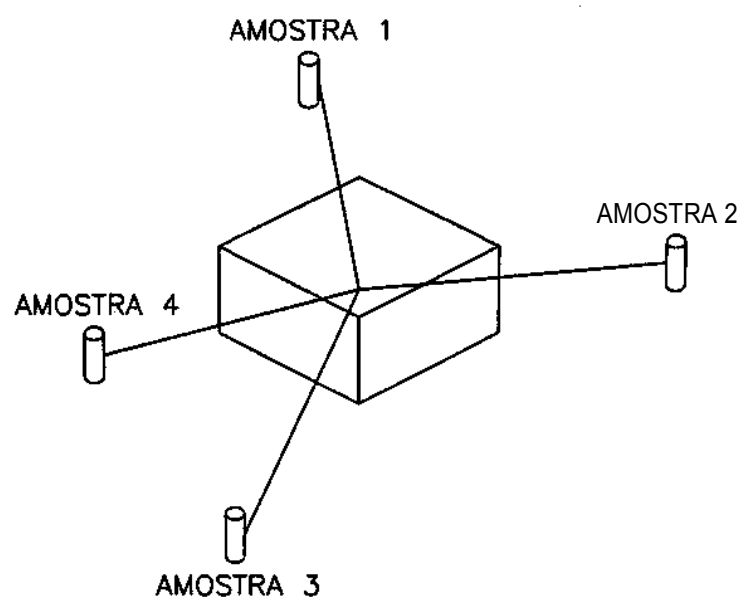

Figura 2 - Avaliação do bloco de cubagem a partir do ponto central.

As equações (7) e (8) são válidas tanto para estimativas pontuais como de blocos, conforme suporte teórico dado pelo Teorema da combinação das estimativas de krigagem (Journel \& Huij̣bregts 1978). Dado um bloco subdividido em nsb sub-blocos as seguintes relações são válidas:

$$
\begin{aligned}
& \gamma\left(x_{i}-x_{o}\right)=\frac{1}{n s b} \sum_{j=1}^{n d} \gamma\left(x_{i}-x_{o, j}\right) \\
& \lambda_{i}=\frac{1}{n s b} \sum_{j=1}^{n s b} \lambda_{i, j} \\
& \mu=\frac{1}{n s b} \sum_{j=1}^{n s b} \mu_{j}
\end{aligned}
$$

Assim, o teor médio do bloco e a variância de krigagem de bloco podem ser determinados como:

$$
Z^{*}\left(x_{0}\right)=\frac{1}{n s b} \sum_{j=1}^{n s b} Z^{*}\left(x_{o . j}\right)
$$


A)
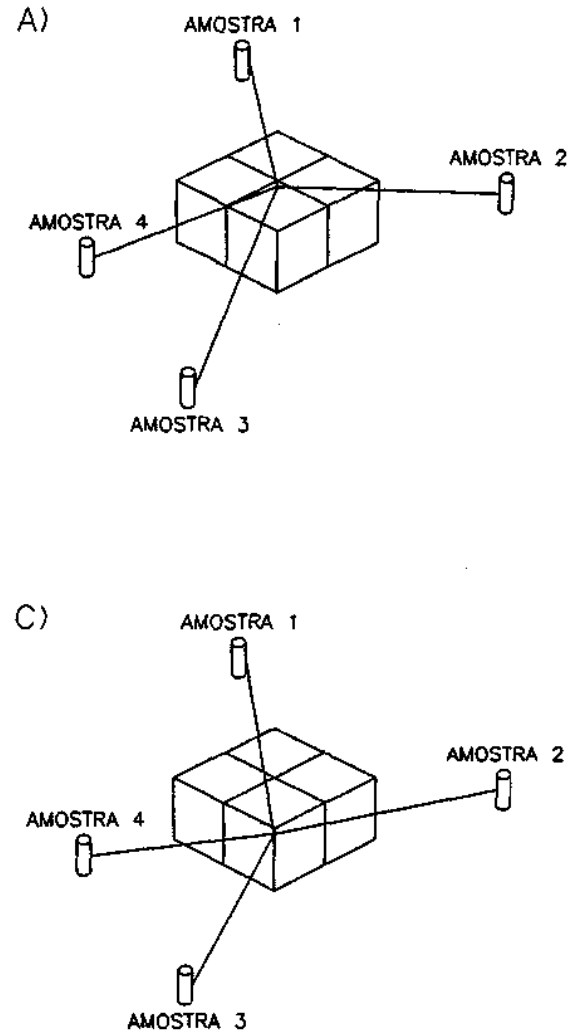

B)
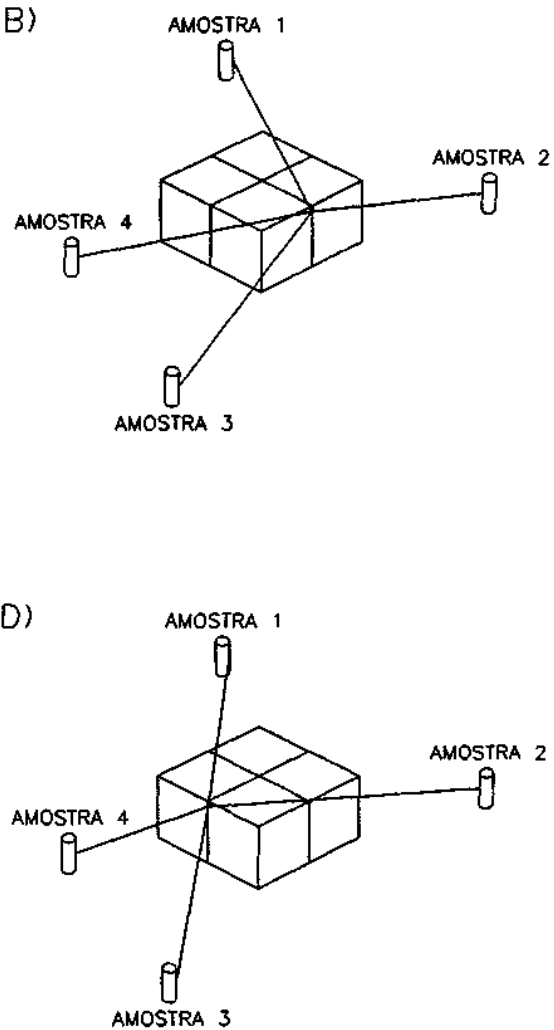

Figura 3 - Avaliação do bloco de cubagem, após sua subdivisão em nsb=4 sub-blocos, os quais são avaliados e combinados de acordo como Teorema da combinação das estimativas de krigagem (Journel \& Huijbregts 1978)

$$
\sigma_{K o}^{2}=\frac{1}{n s b} \sum_{j=1}^{n s b} \sigma_{K o, j}^{2}
$$

onde: $\quad Z^{*}\left(x_{o, j}\right)$ e $\sigma_{K o, j}^{2}=\sum_{i=1}^{n} \lambda_{i, j} \gamma\left(x_{i}-x_{o, j}\right)+\mu_{j}$

são, respectivamente, o valor estimado e sua variância de krigagem associados ao j-ésimo sub-bloco.

Pode-se demonstrar que as expressões ( 8 ou 9 ) e (19) são equivalentes. Substituindo-se (15), (16) e (17) em (9), tem-se:

$$
\sigma_{K O}^{2}=\sum_{i=1}^{n}\left[\left(\frac{1}{n s b} \sum_{j=1}^{n s b} \lambda_{i, j}\right)\left(\frac{1}{n s b} \sum_{j=1}^{n s b} \gamma\left(x_{i}-x_{o, j}\right)\right)+\frac{1}{n s b} \sum_{j=1}^{n s b} \mu_{j}\right],
$$

rearranjando-se os sinais de somatoria:

$$
\sigma_{K o}^{2}=\frac{1}{n s b} \sum_{j=1}^{n s b} \underbrace{\left[\sum_{i=1}^{n} \lambda_{i, j} \gamma\left(x_{i}-x_{o, j}\right)+\mu_{j}\right]}_{\sigma_{k 0 . j}^{2}}
$$

que é exatamente a equação (19).

Da mesma forma, Yamamoto (2000) demonstrou que a variância de interpolação, tal como calculada pela expressão (10), é também válida para a krigagem de bloco. Neste caso, a variância de interpolação associada à estimativa de cada sub-bloco é igual a:

$$
S_{o, j}^{2}=\sum_{i=1}^{n} \lambda_{i, j}\left[Z\left(x_{i}\right)-Z^{*}\left(x_{o, j}\right)\right]^{2}
$$

Conforme o desenvolvimento da equação (10), feito por Yamamoto (2000), a variância de interpolação associada à estimativa de bloco pode ser expressa como:

$$
S_{o}^{2}=\underbrace{\frac{1}{n s b} \sum_{j=1}^{n s b} S_{o, j}^{2}}_{\text {Var.dentro dosblocos }}+\underbrace{\frac{1}{n s b} \sum_{j=1}^{n s b}\left[Z^{*}\left(x_{\sigma_{r}}\right)-Z^{*}\left(x_{o}\right)\right]^{2}}_{\text {Var.entre blocos }}
$$

Esta expressão mostra que a variância de interpolação calculada a partir dos pesos da krigagem ordinária de bloco e igual à média das variâncias de interpolação, determinadas nos nsb sub-blocos mais uma variância entre blocos. E interessante notar que a expressão (10) reconhece os dois suportes (sub-blocos e bloco) de acordo com a relação de aditividade de variâncias de Krige (Isaacks \& Srivastava 1989).

CÁlCULO DO ERRO PARA CLASSIFICAÇÃO DE RESERVAS MINERAIS A variância de estimativa ou variância de krigagem foi proposta como uma medida da incerteza associada à estimativa feita por meio da krigagem ordinária. Contudo, como demonstrado por diversos autores (Journel 1986, Olea 1991, entre outros), a variância de krigagem mede apenas a configuração espacial dos pontos de dados e, por isso, não reconhece a dispersão local dos mesmos. A dispersão local é de importância vital para fins de classificação de reservas, pois discrimina regiões de alta e baixa variabilidade, às quais podem ter atribuídas classes de baixa e alta confiabilidade, respectivamente. A variância de krigagem, ao utilizar a dispersão média do depósito medida por meio do variograma, não consegue discriminar regiões de alta e baixa variabilidade. Por outro lado, a variância de interpolação é uma alternativa que mede a dispersão local, bem como reconhece o efeito proporcional quando existente (distribuições lognormais).

Diversos autores (Froidevaux 1982, Isaacks \& Srivastava 1989, Vallée \& Cote 1992 e Wober \& Morgan 1993) têm proposto calcular o erro com base no intervalo clássico em torno da estimativa, usando a distribuição normal e o desvio padrão da krigagem. Nestas aproximações, a distribuição normal não é adequada, pois o tamanho das amostras em problemas de avaliação de reservas é sempre inferior a 40-60 amostras/bloco avaliado.

Diehl \& David (1982) e Wellmer (1983) propuseram, também com base no desvio padrão da krigagem, utilizar o Teorema do Limite Central para calcular o erro em torno de uma estimativa. De fato, o Teorema do Limite Central proporciona uma boa aproximação para uma distribuição normal. Contudo, estes autores não consideraram o intervalo de confiança para determinação do erro, mas simplesmente o coeficiente de variação multiplicado pelo valor crítico de t, como segue: 


$$
E R R O=\frac{\sigma_{E}}{Z^{*}\left(x_{o}\right)} t_{g l, s, s}
$$

onde ( $\sigma_{\mathrm{E}}$ é o desvio padrão de estimativa no sentido genérico podendo ser substituído pelo desvio padrão de krigagem ou de interpolação, e $\mathrm{t}_{\mathrm{glns}}$ é o valor crítico da distribuição $\mathrm{t}$ de Student para gl graus de liberdade e nível de significância ns.Yamamoto \& Rocha (1996) propuseram a seguinte expressão para o cálculo do erro de estimativa ou tolerância permitida, para fins de classificação de reservas minerais:

$$
E R R O=\frac{1 / 2 I C_{N C(\%)}}{Z^{*}\left(x_{o}\right)}=\frac{\sigma_{E} \cdot t_{g l, n s}}{Z^{*}\left(x_{o}\right) \cdot \sqrt{n}} 100(\%)
$$

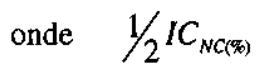

é a metade do intervalo de confiança a um nível de confiança (NC), $\sigma_{\mathrm{E}}$ é o desvio padrão da estimativa, tọlins é o valor crítico de $\mathrm{t}$ para gl graus de liberdade e um nível de significância $n s, Z^{*}\left(\mathrm{x}_{\mathrm{o}}\right)$ é o valor estimado e $n$ o número de amostras utilizadas para a estim $c^{*}$ '. A figura 4 mostra a curva da distribuição t centrada na estimativa $X$ e o seu intervalo de confiança a um determinado nível de confiança. A distribuição t só depende do número de graus de liberdade. Quando o número de graus de liberdade tende ao ${ }^{\circ}$, a distribuição t passa para uma normal. Contudo, a partir de 40-60 amostras, a distribuição t passa praticamente para uma normal. Como dificilmente utiliza-se 40-60 amostras para avaliação de um bloco de cubagem, o erro de uma estimativa deve ser determinado segundo o Teorema do Limite Central (expressão 22) e, portanto, considerando a distribuição.

A expressão (22) tem ser " ${ }^{\prime}$ para cálculo do erro associado a estimativas pontuais, pois te $\sqrt{ } n$ em seu denominador, sendo $\mathrm{n}$ o número de amostras. Contudo, em estin " is de bloco, onde o bloco é su' " lo em nsb sub-blocos, a $\vee n$ deveria ser substituída pela $\sqrt{ } n s D$, pois, na verdade, são utilizados nsb sub-blocos para a estimativa de teor (18) e da variância de interpolação (20). Assim, a expressão para cálculo do erro associado à estimativa de bloco fica:

$$
E R R O=\frac{\sigma_{E} \cdot t_{g l \mu s s}}{Z^{*}\left(x_{0}\right) \sqrt{n s b}} 100(\%)
$$

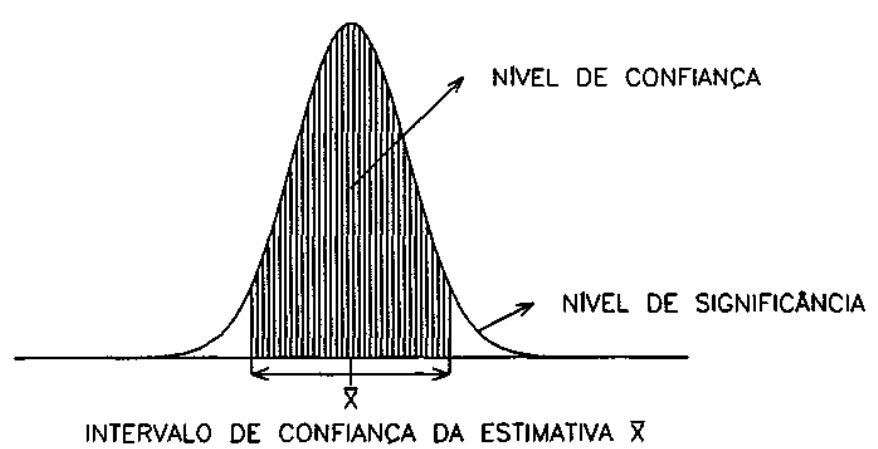

Figura 4 - Curva da distribuição t de St ${ }^{+\cdots}$-nt, utilizada para o cálculo do intervalo de confiança da estimativa $X$ a um determinado nível de confiança NC.

Tabela l - Modelo para classificação de recursos minerais, em comparação com as propostas de Diehl \& David (1982), Wellmer

\begin{tabular}{|lcccc|}
\hline Recursos $\Rightarrow$ & \multicolumn{2}{c}{ Medido } & Indicado & Inferido \\
\hline Reservas $\Rightarrow$ & Provada & Provavel & Possivel & Inferida \\
\hline Diehl \& & Erro: $\pm 10 \%$ & Erro: $\pm 20 \%$ & Erro: $\pm 40 \%$ & Erro: $\pm 60 \%$ \\
David (1982) & N.C. $>\mathbf{8 0 \%}$ & N.C. $.60-80 \%$ & N.C. $40-60 \%$ & N.C. $: 20-40 \%$ \\
\hline Wellmer & Erro: $\pm 10 \%$ & Erro: $\pm 20 \%$ & Erro: $\pm 30 \%$ & Erro: $\pm 50 \%$ \\
(1983) & N.C.: $90 \%$ & N.C. $.90 \%$ & N.C.: $90 \%$ & N.C.: $90 \%$ \\
\hline ONU $\%$ & Erro: $\pm 0-20 \%$ & Erro: $\pm 20-50 \%$ & Ento: $> \pm 50 \%$ \\
DNPM (1992) & N.C.: $95 \%$ & N.C.: $95 \%$ & N.C.: $95 \%$ \\
\hline Este trabalho & Erro: 0-20\% & Erro: $20-50 \%$ & Erro: $>50 \%$ \\
& N.C.: $90 \%$ & N.C.: $90 \%$ & N.C.: $90 \%$ \\
\hline N.C. $=$ é o nível de confiança conforme a Figura 4. & &
\end{tabular}
Segnopolis (PR), conforme o mapa de localização da figura 5.

Segundo Conde \& Yamamoto (1995), a jazida de Canoas 2 é representada por um corpo lenticular, alongado na direção NE-SW, com dimensões aproximadas de $1 \mathrm{~km}$ de extensão (mínima) por 150 $\mathrm{m}$ de largura (maxima) e 6,5 $\mathrm{m}$ de espessura (máxima). Ainda de acordo com estes autores, aproximadamente na parte central, o corpo é interrompido por uma erosão que o divide em dois setores denominados: Canoas 1 (SW do Córrego Pinheirinho) e Canoas 2 (NE do Córrego Pinheirinho). Na Mina de Canoas 2, as principais rochas são quartzitos, xistos anfibolíticos, rochas cálcio-silicáticas, anfibolitos e quartzo-mica xistos, ocorrendo também pequenos diques de metadiabásio (Conde \& Yamamoto 1996). A mineralização ocorre em rochas cálcio-silicáticas, carbonático-baritiferas e, em menor escala, metapelitos, contendo proporções varíaveis de sulfetos (galena e esfalerita), 
formando minérios do tipo disseminado e maciço (Conde \& Yamamoto 1995).

Os dados da Mina de Canoas 2 provêm de 47 furos de sonda rotativa a diamante, totalizando $1724,17 \mathrm{~m}$, com recuperação média em tomo de $95 \%$. As amostras, na zona mineralizada, foram coletadas a intervalos de $0,5 \mathrm{~m}$. Os teores obtidos nos intervalos amostrados foram compostos para a zona mineralizada, definida como aquela contendo teores de $\mathrm{Pb}+\mathrm{Zn}$ iguais ou superiores a $5 \%$, conforme o teor de corte estabelecido pela Plumbum Metalurgia e Mineração S.A., em 1992. Este teor de corte foi considerado para os fins do presente estudo, pois trata-se em testar a metodologia proposta. A figura 6 apresenta os furos de sonda dentro da área mineralizada, bem como a sua subdivisão em blocos de cubagem de $25 \times 25 \mathrm{~m}$.

\section{Avaliação dos recursos de $\mathrm{Pb}$ e $\mathrm{Zn}$ da Mina de Canoas 2}

Os recursos de $\mathrm{Pb}$ e $\mathrm{Zn}$ foram avaliados, conforme os blocos de cubagem (Fig. 6), por meio da técnica da krigagem ordinária de bloco. Para esta finalidade, foram considerados os variogramas teóricos modelados por Conde \& Yamamoto (1996). O modelo para a variável $\mathrm{Pb}$ é descrito por:

$$
\gamma(h)=0,60+2,80\left[1,5 \frac{h}{240}-0,5\left(\frac{h}{240}\right)^{3}\right] \text { para } h<240 m
$$

$$
\gamma(h)=3,40 \text { para } h \geq 240 m
$$

e para a variável $\mathrm{Zn}$ por:

$$
\gamma(h)=1,00+3,30\left[1,5 \frac{h}{200}-0,5\left(\frac{h}{200}\right)^{3}\right] \text { para } h<200 \mathrm{~m}
$$

\section{$\gamma(h)=4,30$ para $h \geq 200 m$}

Os blocos de cubagem foram avaliados por krigagem ordinária, conforme os variogramas descritos em (24) e (25), usando as informações dos oito pontos vizinhos próximos, selecionados pelo critério dos quadrantes. Na realidade, além do teor médio do bloco, interessam as variâncias de krigagem e de interpolação, as quais serão utilizadas para o cálculo dos erros de tolerância para classificação de recursos.

Análise comparativa das variâncias de krigagem e de interpolacão Com o objetivo de aferir as propriedades das variâncias de krigagem e de interpolação, foi realizada uma análise comparativa, através de diagramas de dispersão. Ao invés de variâncias de krigagem e de interpolação foram considerados os respectivos desvios padrão, pois doravante eles serão utilizados para cálculo do erro de tolerância. Os diagramas de dispersão foram construídos lançando-se no eixo das ordenadas o desvio padrão e no eixo das abscissas o teor. Os resultados obtidos para a variável $\mathrm{Pb}$ encontram-se na figura $7 \mathrm{e}$ para a variável $\mathrm{Zn}$ na figura 8 .

Embora as correlações não sejam significativamente altas, pode-se concluir que o desvio padrão de interpolação é proporcional ao teor médio do bloco, confirmando que pode reconhecer o efeito proporcional quando existente. Por outro lado, o desvio padrão de krigagem mostra uma relação inversa com o teor médio do bloco, mostrando que não reconhece o efeito proporcional e independe dos valores e dispersão locais. Portanto, o desvio padrão de interpolação aproxima melhor a incerteza associada à estimativa que a tradicional medida proporcionada pela krigagem.

Classificação dos recursos de $\mathrm{Pb}$ e $\mathrm{Zn}$ da Mina de Canoas 2 Com os dados de teor e desvios padrão foram calculados os erros de tolerância, a um nível de significância igual a $90 \%$, usando tanto o desvio padrão de krigagem como o de interpolação, de acordo com a expressão geral (23). Os erros assim obtidos foram classificados conforme a proposta apresentada neste artigo (Tabela 1).

A figura 9 apresenta os resultados da classificação dos recursos de $\mathrm{Pb}$ e a figura 10 os mesmos para os de $\mathrm{Zn}$.

Como se pode observar pelos resultados obtidos, não há uma boa correlação entre as classificações. Para a variável $\mathrm{Pb}$, apenas 20 blocos apresentaram a mesma classificação: 12 blocos de recurso indicado e 8 blocos de recurso inferido, enquanto para a variável $\mathrm{Zn}$, apenas 12 blocos tiveram a mesma classificação, com 7 blocos como recurso indicado e 5 como recurso inferido. Em geral, verifica-se que o desvio padrão de krigagem é superestimado em relação ao desvio padrão de interpolação, como se pode constatar na Tabela 2. Portanto, há uma tendência do desvio padrão de krigagem classificar os recursos em

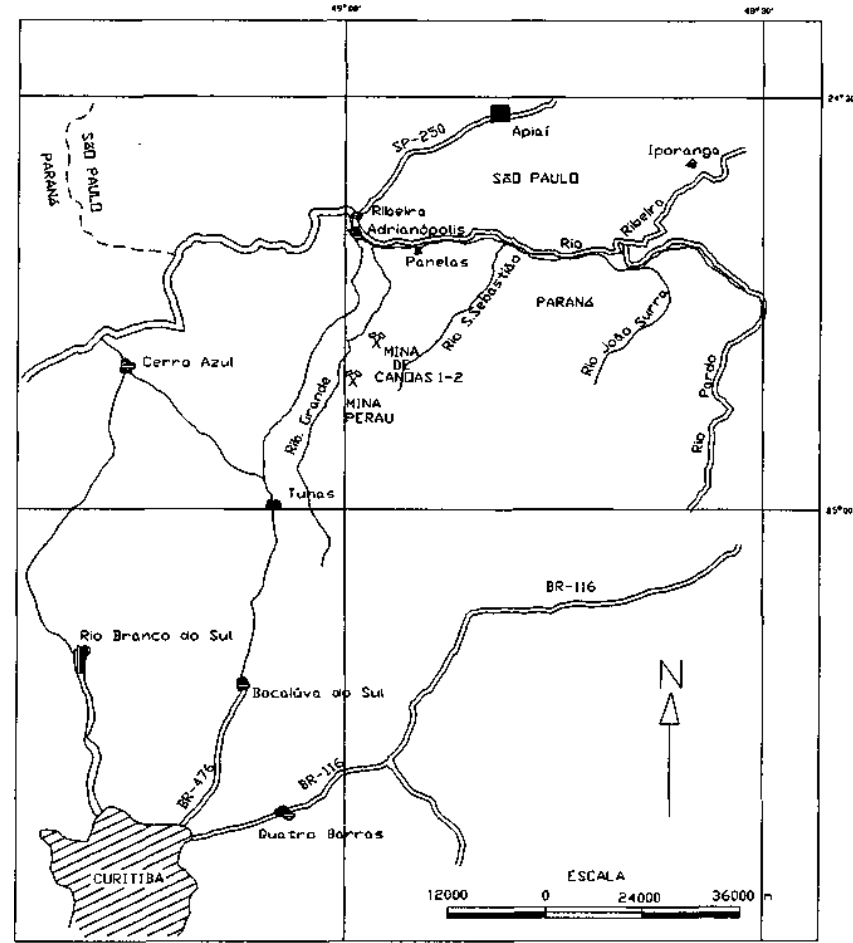

Figura 5 - Mapa de localização da Mina de Canoas 2 (Conde \& Yamamoto 1996).

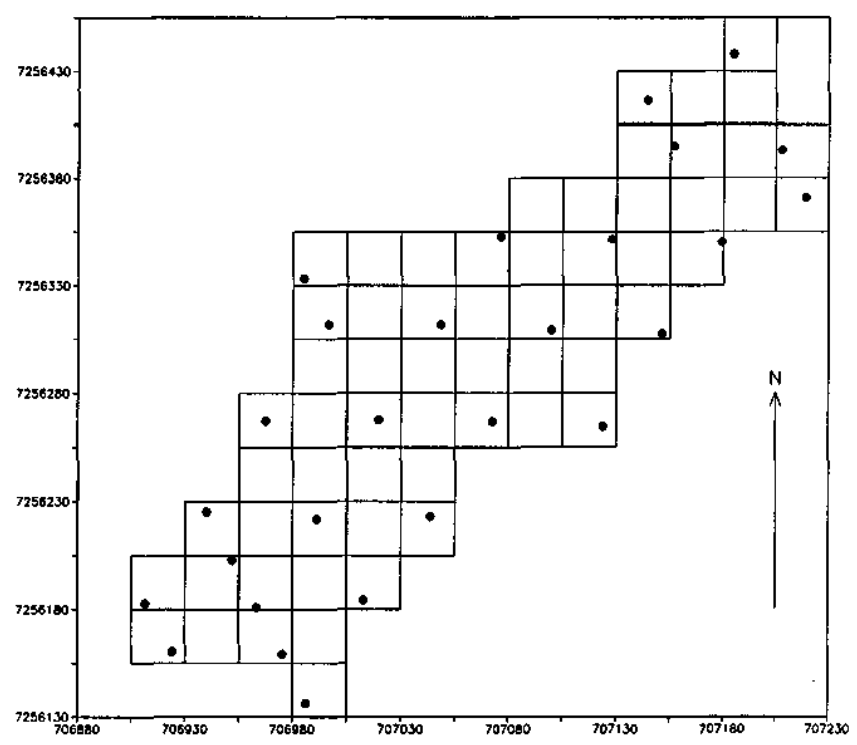

Figura 6 - Mapa de localização dos furos da sonda e delimitação da área mineralizada e sua subdivisão em blocos e cubagem.

Tabela 2 • Distribuição dos blocos (\%) conforme a classificação de recursos, usando os desvios padrão de krígagem e de interpolação.

\begin{tabular}{lcccc}
\hline & \multicolumn{2}{c}{$\mathrm{Pb}$} & \multicolumn{2}{c}{$\mathrm{Zn}$} \\
\hline & $\sigma_{\mathrm{KO}}$ & $\mathrm{S}_{0}$ & $\sigma_{\mathrm{KO}}$ & $\mathrm{S}_{0}$ \\
\hline Medido & 0 & 3,5 & 0 & 5,2 \\
\hline Indicado & 24,1 & 79,3 & 15,5 & 84,5 \\
\hline Inferido & 75,9 & 17,2 & 84,5 & 10,3 \\
\hline
\end{tabular}


A

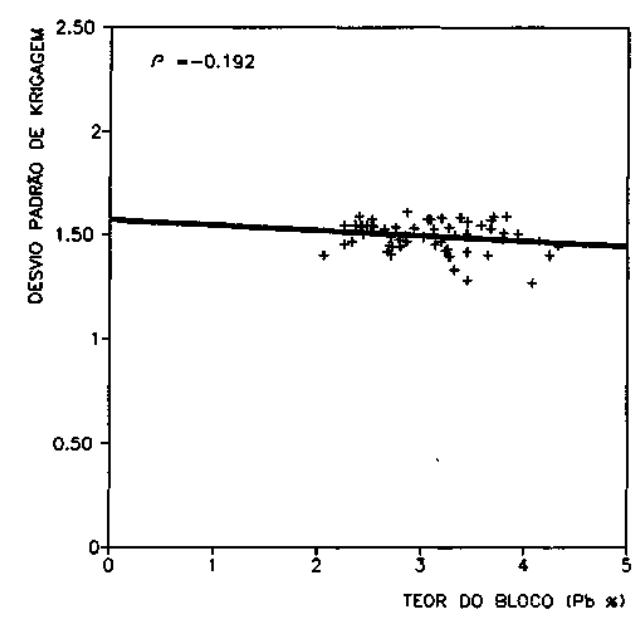

B

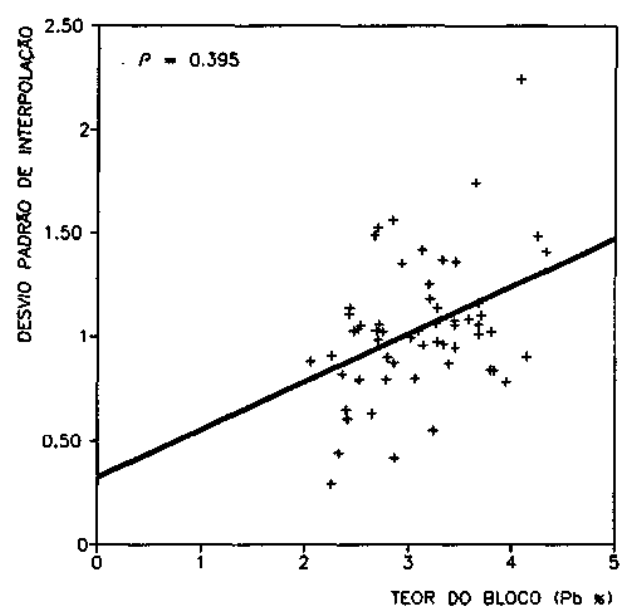

Figura 7 - Diagramas de dispersão para a variável $\mathrm{Pb}$, do desvio padrão de krigagem em função do teor do bloco (A) e do desvio padrão de interpolação contra o teor do bloco (B).

A

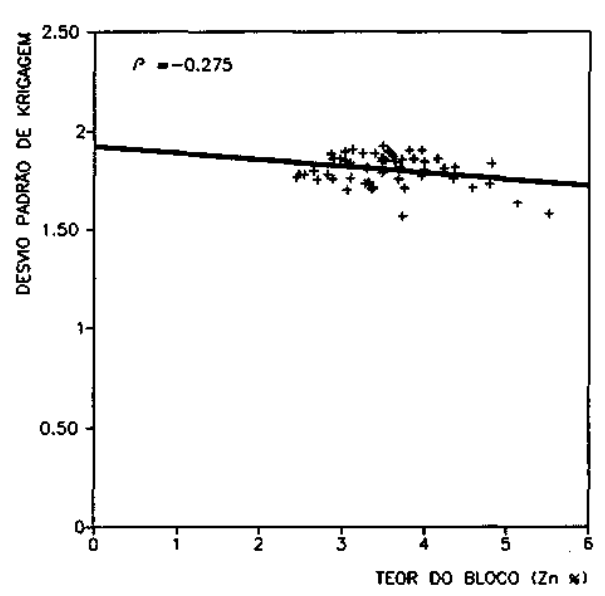

B

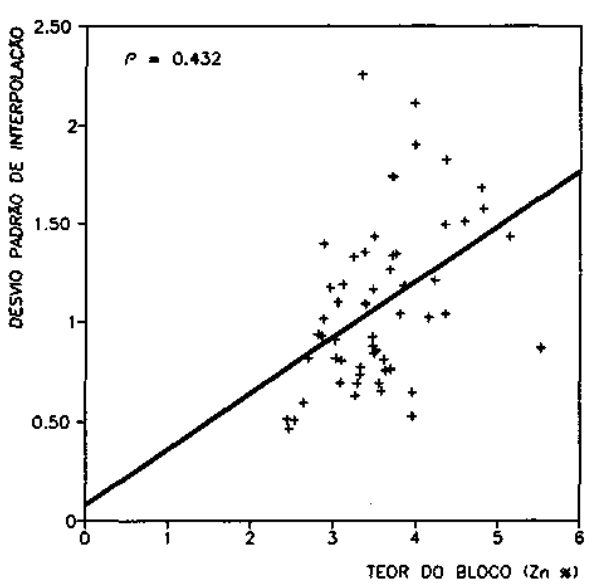

Figura 8 - Diagramas de dispersão para a variável Zn, do desvio padrão de krigagem em função do teor do bloco (A) e do desvio padrão de interpolação contra o teor do bloco (B).
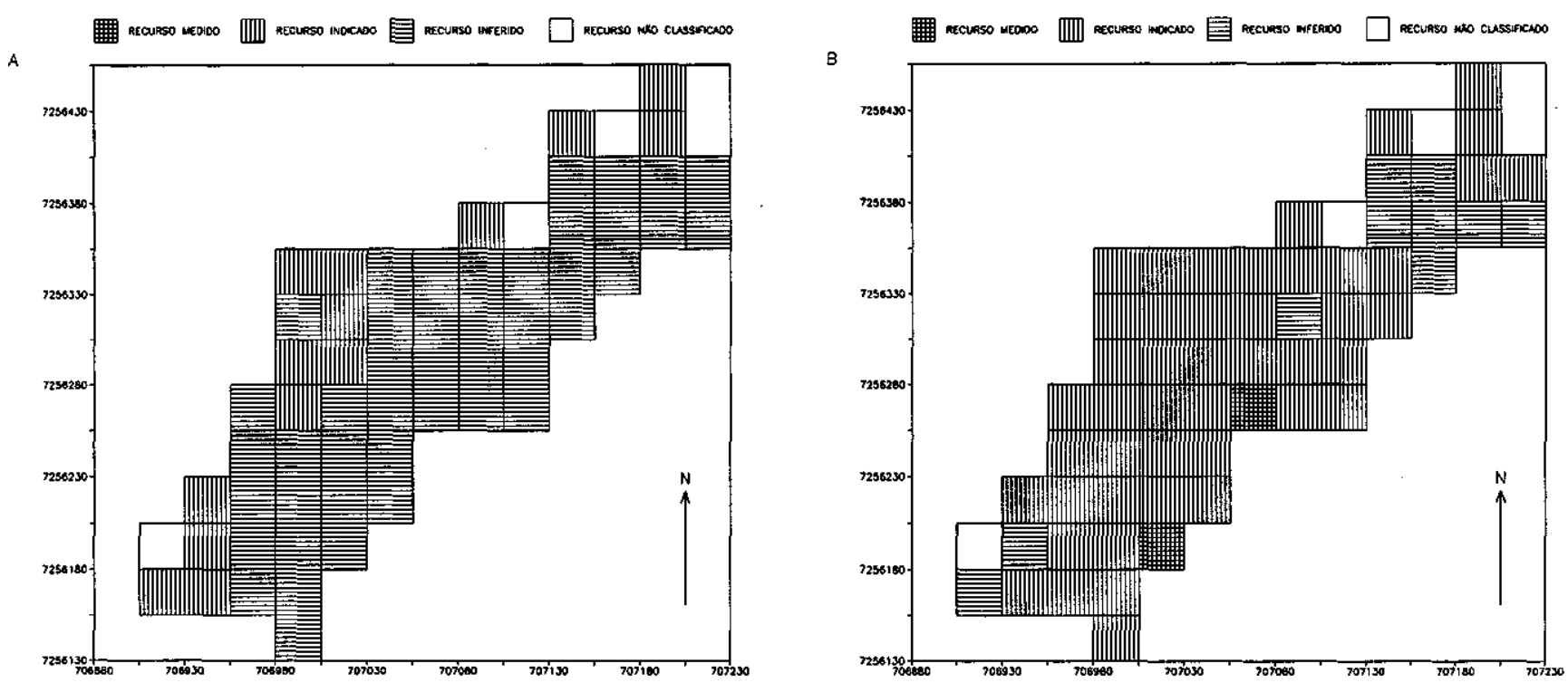

Figura 9 - Classificação dos recursos de Pb usando o desvio padrão de krigagem (A) e desvio padrão de interpolação (B). 

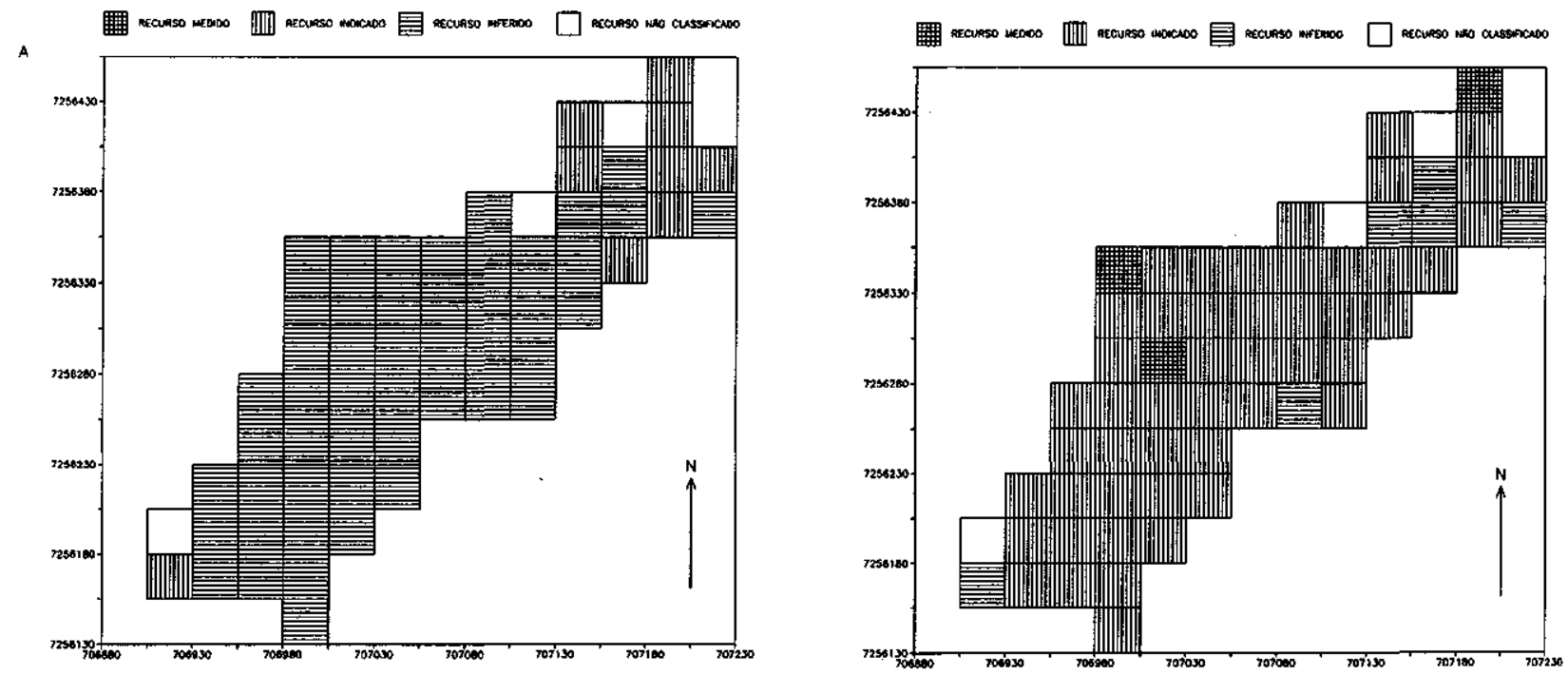

Figura W- Classificação dos recursos de Zn usando o desvio padrão de krigagem (A) e desvio padrão de interpolação (B).

classes de menor confiabilidade (recurso inferido: $75,9 \%$ para o $\mathrm{Pb}$ e $84,5 \%$ para o $\mathrm{Zn}$ ), do que o desvio padrão de interpolação (recurso indicado: $79,3 \%$ para o $\mathrm{Pb}$ e $84,5 \%$ para o $\mathrm{Zn}$ ). Contudo, considerando as dimensões do depósito, a densidade de amostragem e a sua pequena variabilidade natural, é razoável supor que a maioria dos blocos sejam classificados ao menos como indicados e não como inferidos. Assim, pode-se concluir que o desvio padrão de interpolação, ao medir a dispersão local dos dados e reconhecer o efeito proporcional, permite classificar os recursos minerais com maior precisão que o desvio padrão de krigagem.

CONSIDERAÇÕES FINAIS Como ficou demonstrado neste trabalho, a variância de interpolacão pode quantificar com precisão a incerteza associada à estimativa da krigagem ordinária, à medida que leva em consideração a dispersão local dos pontos de dados e, assim, reconhece o efeito proporcional. Por outro lado, a variância de krigagem ordinária não apresenta as mesmas características e, por isso, não pode ser utilizada para quantificação da incerteza e muito menos para fins de classificação de recursos minerais, como foi salientado por

\section{Referências}

Armstrong M. 1994. Is research in mining geostats as dead as a dodo ? in: Dimitrakopoulos R. (ed.), Geostatisticsfor the next century: Kluwer Academic Publishers, Dordrecht, p. 303-312.

Australasian Institute of Mining and Metallurgy-AIMM. 1996. Australasian code for reporting of identified mineral resources and ore reserves. The AusIMM Bulletin (4): $1-19$.

Barnes R.P. \& Johnson T.B. 1984. Positive kriging. In: Verly et al (eds) Geostatisticsfor natural resources characterization. Reidel. Part I, p.231-244.

Conde R.P. \& Yamamoto J.K. 1995. Avaliação de reservas por métodos convencionais: um estudo de caso na Mina de Canoas 2 (PR). Boi. IG-USP, Ser. Cient., 26:13-28.

Conde R.P. \& Yamamoto J.K. 1996. Avaliação de reservas por métodos computacionais: um estudo de caso na Mina de Canoas 2 (PR). Rev. Brás. Geoc., 26:35-42.

Departamento Nacional da Produção Mineral-DNPM. 1992. Bases técnicas de um sistema de quantificação do património mineral brasileiro. Brasília, DNPM. 28p. (Relatório do Grupo de Trabalho, Portaria no 03 de 24/10/90, da Secretaria Nacional de Minas e Metalurgia).

Deutsch C.V. 1996. Correcting for negative weights in ordinary kriging: Computers \& Geosciences, 22:765-773.

Diehl P. \& David M. 1982. Classification of ore reserves/resources based on geostatistical methods. CIM Buli., 75(838): 127-136.

Froidevaux R. 1982. Geostatistics and ore reserve Classification. CIM Buli. 75(843):77-83.

Froidevaux R. 1993. Constrained kriging as an estimator of local distribution functions./n: Capasso V., Girone G., Posa D. (eds.) Statistics of spatial processes: theory and applications, p.106-118.

Herzfeld U.C. 1989. A note on programs performing kriging with nonnegative weights. Math. Geoi, 21:391-393.

Isaacks E.H. \& Srivastava R.M. 1989. An introduction to applied geostatistics: Oxford University Press, New York, 561 p.

Journel A.G. 1986. Geostatistics: models and tools for the Earth Sciences. Math. Geol., 18:119-140.

Journel A.G. \& Huijbregts C.J. 1978. Mining geostatistics. Academic Press, London, 600p.
Armstrong (1994). Desse modo, a variância de interpolação pode ser utilizada para quantificar o erro de tolerância em torno da estimativa para classificacãa de recursos minerais. A proposta apresentada para classificação de recursos minerais foi baseada em classes de certeza adotados pela ONU (apud Valente 1980), porém com os erros de tolerância calculados sob um nível de confiança igual a 90\%, que é compatível com materiais geológicos. As aplicações da metodologia proposta, para classificação dos recursos de $\mathrm{Pb}$ e $\mathrm{Zn}$ na Mina de Canoas 2, comprovaram a eficiência da variância de interpolação em relação à variância de krigagem.

Agradecimentos À Fundação de Amparo à Pesquisa do Estado de São Paulo - FAPESP (Processo 92/1460-7), cujo apoio financeiro permitiu a realização dos trabalhos no Depósito de Canoas. O Autor Sénior agradece ao Conselho Nacional de Desenvolvimento Científico e Tecnológico-CNPq (Processo 304612/89-8) pelo apoio financeiro que permitiu a realização deste trabalho. A dois revisores anónimos da RBG pelas sugestões.

Journel A.G. \& Rao S.E. 1996. Deriving conditional distributions from ordinary kriging: Stanford Center for Reservoir Forecastíng, Stanford, 25p. (Report \#9)

Koch Jr. G.S. \& Link R.F. 1971. Statistical analysis ofgeological data. Dover Publications, New York. v. 1,375p.

Matheron G. 1971. The theory of regionalized variables and its applications. Fontainebleau, É cole Nationale Supérieux das Mines de Paris. 21 Ip.

Olea R.A. 1991. Geostatistical glossary and multilingual dictionary. Oxford University Press, New York. 175p.

Popoff C.C. 1966. Computing reserves of mineral deposits: principies and conventional methods. Washington, Bureau of Mines. 113p. (I.C. 8283).

Valente J. 1980, Avaliação geoestatística com parametrização da jazida fosfática de Anitápolis. In: SBG, Congresso Brasileiro de Geologia, 31, Camboriú, 1980, Anais..., v.2, p,1243-1257.

Vallée M. \& Cote D. 1992. The guide to the evaluation of gold deposits: integrating deposit evaluation and reserve inventory practices. CIM Buli., 85(957):50-61.

Wellmer F.W. 1983. Classification of ore reserves by geoestatistical methods. Enmetall, 36:315-321.

Wober H.H. \& Morgan P. J. 1993 Classification of ore reserves based on geostatistical and economic parameters. CIM Bulletin, 86(966):73-76.

Yamamoto J. K. 1991. Comparação de métodos computacionais para avaliação de reservas: um estudo de caso na Jazida de Cobre de Chapada, GO. Universidade de São Paulo. 175p. (Tese de doutoramento)

Yamamoto J.K. 2000. An alternative measure of the reliability of ordinary kriging estimates. Math. Geol., 32:489-509..

Yamamoto J.K. \& Rocha M.M. 1996. Revisão e recomendações para o cálculo e classificação de reservas minerais. Rev. Bros. Geoc., 26:243-254.

Manuscrito A-1060 Recebido em 05 de dezembro de 1998 Revisão dos autores em 25 de agosto de 1999 Revisão aceita em 26 de agosto de 1999 